\title{
Femtosecond multiple-pulse impulsive stimulated Raman scattering spectroscopy
}

\author{
A. M. Weiner and D. E. Leaird \\ Bellcore, Red Bank, New Jersey 07701-7040
}

Gary P. Wiederrecht and Keith A. Nelson

Department of Chemistry, Massachusetts Institute of Technology, Cambridge, Massachusetts 02139

Received August 8, 1990; accepted November 5, 1990

\begin{abstract}
Optical control of elementary molecular motion through impulsive stimulated Raman scattering is enhanced by means of timed sequences of femtosecond pulses that are produced by pulse-shaping techniques. In particular, terahertz-rate trains of femtosecond pulses are used for repetitive impulsive excitation of individual phonon modes in an $\alpha$-perylene molecular crystal. When the pulse repetition rate is matched to the desired phonon frequency, mode-selective vibrational amplification is achieved. A comparison of data acquired with the transient-grating and the pump-probe experimental geometries reveals the timing dynamics of the induced phonon oscillations with respect to the driving femtosecond pulse sequence.
\end{abstract}

\section{INTRODUCTION}

Ongoing advances in ultrafast laser technology have led to the generation of optical pulses whose duration is shorter than the time required for many nuclear motions. Spectroscopic use of such pulses has made possible direct timeresolved observations of elementary microscopic motions, such as collective vibrations of crystal lattices and molecular vibrations and rotations, as well as chemical and physical transformations that involve these motions. ${ }^{1-6}$ Increasing attention is now being turned toward not only observation but also control of molecular and collective motion, often with the goals of reaching unusual nonequilibrium states and facilitating chemical or structural changes that may not occur under ordinary conditions. ${ }^{7,8}$ With recently developed techniques for shaping ultrashort laser pulses, ${ }^{9-11}$ production of optical pulse sequences and wave forms that are specially tailored to exercise such control is becoming feasible. In this paper we report an initial application of specially shaped femtosecond pulse trains that achieves improved control over elementary molecular motions. In particular, we demonstrate the use of timed sequences of femtosecond pulses to drive selected vibrations repetitively in an organic crystal in a manner that is closely analogous to the way a child on a swing may be repetitively pushed to reach an increased amplitude of oscillatory motion. ${ }^{12}$

In our experiments we use impulsive stimulated Raman scattering (ISRS), a spectroscopic technique in which an ultrashort laser pulse initiates coherent vibrational motion by exerting a sudden (impulse) driving force on Ramanactive vibrational modes. For ISRS to occur, the pulse duration must be short compared with the vibrational oscillation cycle, The uncertainty-limited spectral band= width of the pulse then exceeds the vibrational frequency, and stimulated Raman scattering can occur through mixing among frequency components contained within the bandwidth. ISRS is a completely time-domain analog to the more usual frequency-domain coherent Raman excitation techniques, in which the frequency difference between two tunable lasers is adjusted to match the vibrational frequency. One advantage of the time-domain approach is the ability to permit direct observation of timedependent phonon oscillations on a femtosecond time scale. Thus ISRS provides a mechanism through which femtosecond pulses can be used to influence and to observe elementary lattice and molecular motion. ${ }^{2}$

To date, however, the extent of control over nuclear motion has been limited. The coherent vibrational response that is driven by ISRS is usually small, for example, of the order of $10^{-4} \AA$ for molecular displacements in translational optic phonon modes of crystalline solids. Far larger coherent vibrational amplitudes could be driven by readily available femtosecond pulses were it not for the sample damage that would occur at the high peak intensities required. Furthermore, the degree of mode selectivity possible with ISRS is limited. Many Raman-active modes may respond simultaneously to the impulse force that is exerted by a sufficiently short pulse.

We achieved improved control over molecular motion by using terahertz-repetition-rate trains of femtosecond pulses, generated by using femtosecond-pulse-shaping techniques, for repetitive impulsive excitation in ISRS experiments. In particular, by matching the pulse repetition rate to the vibrational frequency, we demonstrated selective amplification of individual phonon modes in an $\alpha$-perylene molecular crystal. ${ }^{12}$ This crystal is of special interest in connection with an excited-state dimerization (actually excimer formation) reaction that may be phonon assisted. ${ }^{13}$ In the ground state the planar perylene molecules align in sandwich pairs; on optical absorption two molecules in a pair move closer together along a path that is believed to coincide to a substantial degree with a 104$\mathrm{cm}^{-1}$ translational optic phonon mode. ${ }^{14}$ Single-pulse 
(A)

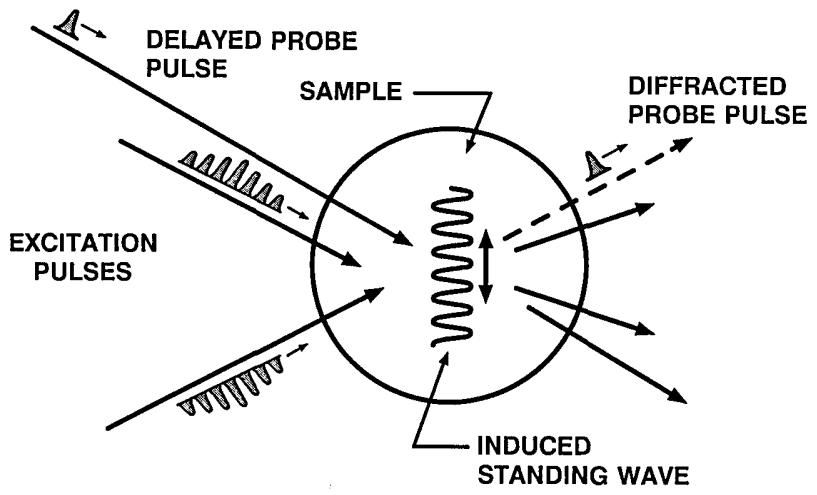

(B)

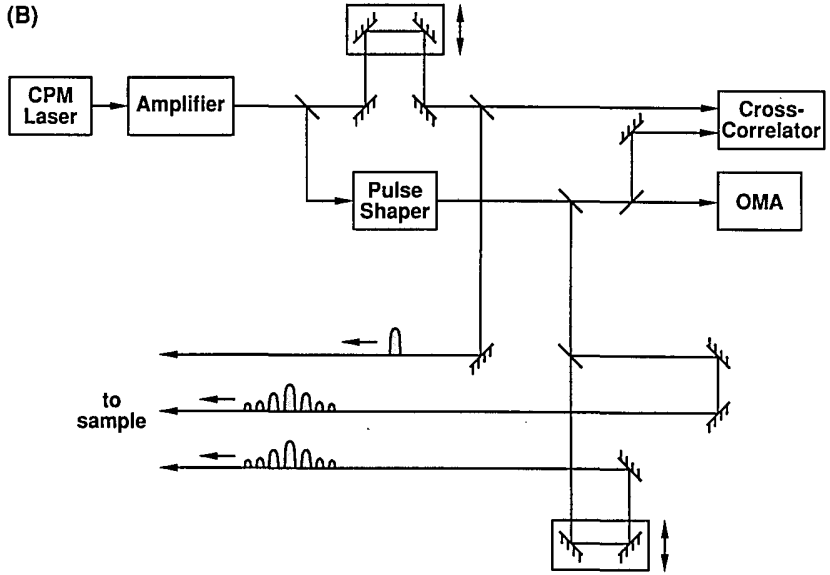

Fig. 1. Experimental arrangement for multiple-pulse ISRS. (A) Sketch of the transient-grating scattering geometry. (B) Block diagram of the optical setup. CPM

ISRS experiments with $\alpha$-perylene crystals were reported previously. ${ }^{15}$ In those experiments simultaneous excitation of the 104- $\mathrm{cm}^{-1}$ mode, together with low-frequency librational modes at 33,56 , and $80 \mathrm{~cm}^{-1}$, was observed. As we shall see, multiple-pulse ISRS makes possible a degree of mode selectivity that is not available with singlepulse ISRS and opens the way for increased vibrational amplitudes.

The remainder of this paper is structured as follows. First we describe the experimental setup and briefly review the femtosecond-pulse-shaping apparatus that makes these experiments possible. We then discuss ISRS data taken in the transient-grating geometry. Single-pulse ISRS data are presented in order to illustrate some of the limitations of this technique. Multiple-pulse ISRS data, which clearly demonstrate mode selectivity and vibrational amplification, are then presented and discussed in detail. We also discuss detuning effects that occur when the pulse repetition rate does not precisely match the vibrational frequency. We further report multiple-pulse ISRS experiments performed in the forward or pumpprobe geometry, in which optical spectral shifts induced by the coherent vibrational motion are detected. These measurements elucidate the timing of the coherent vibrational motion relative to the excitation-pulse sequence that drives it. Finally, we discuss further prospects for multiple-pulse ISRS spectroscopy; in Appendix A we comment on the quantum-mechanical interpretation of our experiments.

\section{EXPERIMENTAL SETUP}

We have performed multiple-pulse ISRS measurements in both transient-grating and pump-probe experimental geometries. The experimental arrangement for our transient-grating measurements is depicted in Fig. 1 (see Section 5 for details of the pump-probe measurements). The source of femtosecond pulses was a colliding-pulse mode-locked (CPM) ring dye laser ${ }^{16}$ and a copper-vapor laser-pumped dye amplifier (OMA) system, ${ }^{17}$ which provided 75 -fs pulses at a $0.62-\mu \mathrm{m}$ wavelength with a pulse energy of several microjoules and an $8.6-\mathrm{kHz}$ repetition rate. A small portion of the output from the amplifier was split off to serve as the probe beam. The remainder of the amplifier output was passed through the pulse-shaping apparatus (described below), where it was converted into a terahertz-rate pulse sequence, and then split to yield two identical excitation beams. All three beams were focused to a common spot in the sample, a 1-mm-thick crystal of $\alpha$-perylene molecular crystal. The $\alpha$-perylene was purified by recrystallization and extensive zone refining, and single crystals were grown from the melt by the Bridgman method. The sample was mounted on a cold finger and cooled to temperatures as low as $5 \mathrm{~K}$. The absorption edge of $\alpha$-perylene occurs near $480 \mathrm{~nm}$, and therefore the perylene is transparent to the $620-\mathrm{nm}$ laser light. The sample was cleaved along the $a b$ crystallographic plane. All the beams were linearly polarized, with all the polarization directions parallel to each other and to either the $a$ or the $b$ crystallographic axis. The energies of the excitation and the probe beams were typically 200 and $35 \mathrm{~nJ}$, respectively. The beams were crossed in a noncoplanar, phase-matched geometry, with a full angle of $\sim 5^{\circ}$ between them. The diameters of the focused pump and probe beams were each $\sim 90 \mu \mathrm{m}$. The relative delay between the two excitation beams was set to zero so that the beams interfere in the sample to produce a standing-wave pattern of coherent vibrational excitation through ISRS. The time-dependent vibrational response was monitored by measuring the coherent scattering (or diffraction) intensity of the temporally delayed probe pulse from the standing-wave grating. For data acquisition the probe beam was chopped, and the diffraction intensity was measured with a power meter and a lock-in amplifier. The probe delay was scanned under computer control by using a stepper-motor-driven translation stage, and the output of the lock-in amplifier was digitized and stored for each setting of the probe delay. In a typical measurement a single scan was accomplished in $\sim 1 \mathrm{~min}$, and five such scans were averaged on the computer.

The terahertz-rate pulse trains used as the excitation beams were generated by means of Fourier-transform pulse shaping. As described previously, ${ }^{9}$ pulse shaping was achieved by passing a single femtosecond pulse through a simple optical system that consists of two gratings, two lenses, and a spatially varying phase mask. The gratings were placed at the (outside) focal planes of the lenses, which were set up as a unit-magnification telescope, and the mask was placed midway between the lenses. After the first grating and lens spatially separated the various optical frequency components contained within the incident ultrashort pulse, the mask altered the relative phases of the dispersed frequency components. 


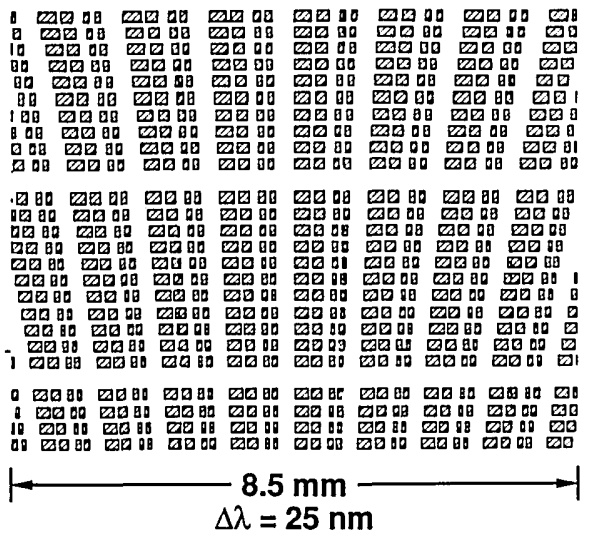

Fig. 2. Layout of a phase mask, designed to produce pulse trains with repetition rates ranging from 340 to $460 \mathrm{fs}$. Each individual pattern (from left to right) corresponds to a particular repetition rate. The repetition period is $340 \mathrm{fs}$ at the top and increases by $5 \mathrm{fs}$ per pattern toward the bottom. The shaded regions in the figure correspond to areas that are etched during the fabrication process (to a depth corresponding to a phase change of approximately $\pi$ ). The unshaded regions are not etched during fabrication.

(In general the mask can alter both phase and amplitude, ${ }^{9,18}$ but in the present investigation we use pure phase masking for high efficiency.) The second lens and grating recombined the optical frequency components into a single collimated beam. The result was a shaped pulse with a temporal profile that is given by the Fourier transform of the pattern transferred by the mask onto the spectrum. The intensity profile of the shaped pulse was measured by cross correlation, with 75 -fs pulses directly from the amplifier as the probe, and the spectral profile of the shaped pulses was measured with a $0.32-\mathrm{m}$ spectrometer and a photodiode array.

In the current experiment the phase was patterned according to periodic repetitions of a pseudorandom binary phase code called an $M$ sequence. The details of the mask design are given elsewhere. ${ }^{19}$ In brief, the spatial periodicity of the phase mask imparts a periodic modulation to the optical spectrum, and this gives rise to a terahertzrate pulse train, with the pulse repetition rate equal to the inverse of the spectral modulation frequency. The phase masks are fabricated on 0.5-in- (1.27-cm-) diameter fusedsilica substrates with standard microlithography and reactive-ion etching techniques. ${ }^{9}$ A layout of one of the masks is shown in Fig. 2. This mask contains approximately 25 distinct phase patterns, each with a different spatial periodicity that is designed to produce a pulse train with a different repetition rate. The phase patterns shown produce sequences that consist of approximately 15 evenly spaced pulses under a smooth envelope, with repetition periods ranging from $340 \mathrm{fs}$ (top) to $460 \mathrm{fs}$ (bottom), selectable in 5-fs increments. The desired pulse sequence is selected by translating the appropriate pattern into the beam path. Other masks were also fabricated to cover the ranges $170-245,245-350$, and $460-700$ fs. Thus essentially any pulse repetition rate that is needed to match phonons in the range $1.4-6.0 \mathrm{THz}\left(50-200 \mathrm{~cm}^{-1}\right)$ could be selected. We note that more complex pulse sequences, including squared-off envelopes of pulses, have also been produced, ${ }^{19}$ but these were not used in the present experiments.

\section{SINGLE-PULSE ISRS}

As a control we recorded ISRS data from perylene excited by a single pair of (unshaped) excitation pulses. The data, shown in Fig. 3 for pulses polarized parallel to the $b$ axis, are similar to those published previously. ${ }^{15}$ The signal consists of a sharp peak at $t=0$, arising from the essentially instantaneous nonlinearity that is associated with distortion of the electronic wave functions, followed by a signal that is due to optic phonons excited through ISRS. Two points are of note. First, the intensity of the ISRS signal is only $\sim 4 \%$ of that arising from the electronic scattering peak, which provides an internal intensity calibration. Second, the ISRS diffraction intensity presents an irregular pattern that is caused by beating among several simultaneously excited phonon modes. A Fourier transform of the scattering data is shown as the solid curve in Fig. 4. Because the scattering signal is proportional to the square of the time-dependent refractive-index change induced by the phonons, the Fourier transform consists of a complicated series of lines at frequencies corresponding to sum and difference frequencies of the known perylene modes at 33, 56, 80, and $104 \mathrm{~cm}^{-1}$. Lines are also evident at some of the fundamental phonon frequencies (e.g., at $80 \mathrm{~cm}^{-1}$ ) because of mixing with a nonoscillatory component of the signal arising from electronic excited states generated through two-photon absorption. As a comparison, the dashed curve in Fig. 4 shows the Fourier transform of the main, electronic scattering peak in the data. This curve represents the instrumental response in our experiments, showing that with 75 -fs pulses the ability to excite and monitor coherent vibrational motion rolls off for frequencies above $\sim 3 \mathrm{THz}\left(100 \mathrm{~cm}^{-1}\right)$. Despite this rolloff, the data show well-resolved features out to $5.5 \mathrm{THz}$. Similar data are observed with the polarizations parallel to the $a$ crystallographic axis, although the details of the oscillatory ISRS signal are altered because the various modes are excited with different relative amplitudes. In both cases it is difficult to work backward from the ISRS data to determine which phonons are excited and with what strength.

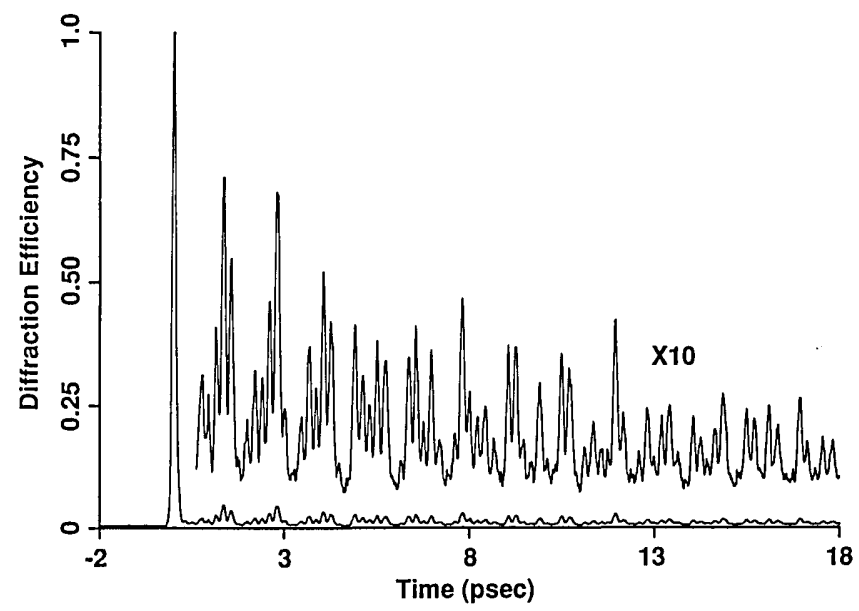

Fig. 3. Single-pulse ISRS data recorded from $\alpha$-perylene with $T<10 \mathrm{~K}$ and with all pulses linearly polarized parallel to the $b$ crystallographic axis. The femtosecond excitation pulses drive several phonon modes whose combined response yields a characteristic beating pattern. The spike at $t=0$ is a purely electronic response of the crystal to the excitation pulses. 


\section{Frequency $\left(\mathrm{cm}^{-1}\right)$}

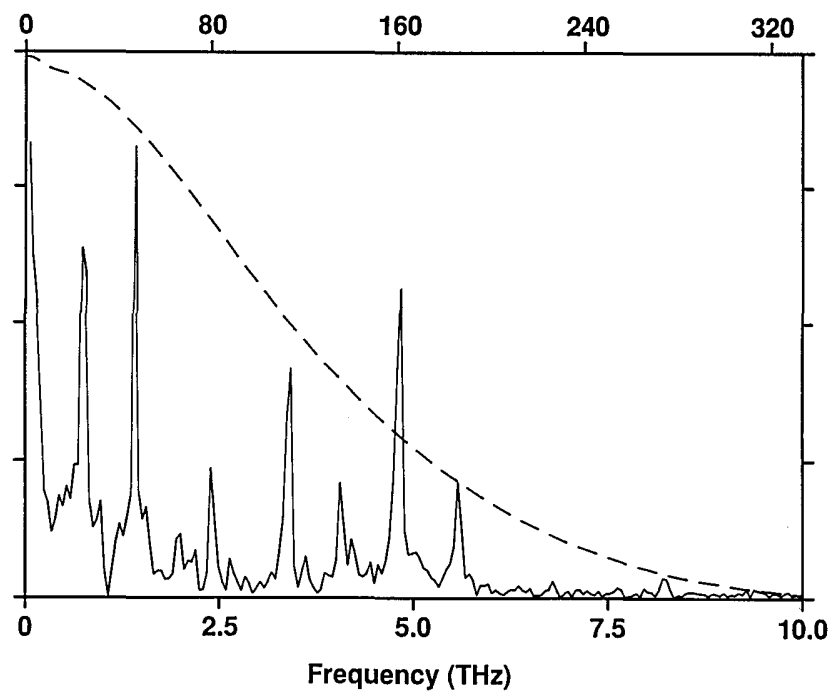

Fig. 4. Solid curve: Fourier transform of the scattering data shown in Fig. 3. The electronic scattering peak at $t=0$ in the time-domain data was suppressed before the Fourier transform was performed. Dashed curve: Fourier transform of the electronic scattering peak, representing the instrumental response function.

(A)

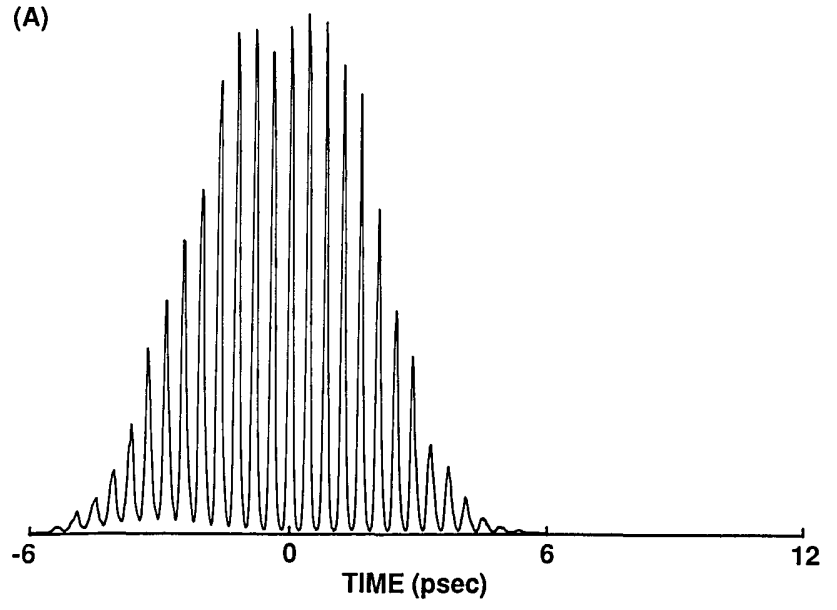

(B)

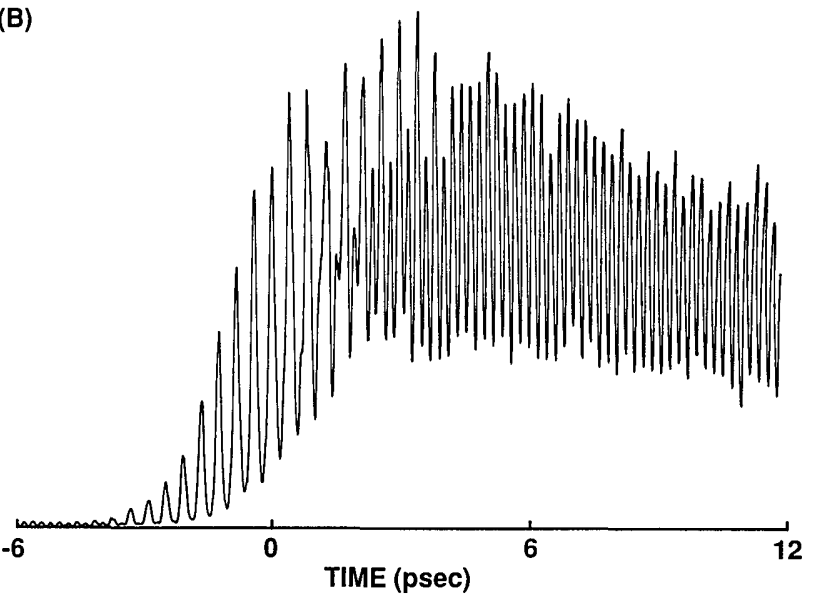

\section{MULTIPLE-PULSE ISRS}

The data in Figs. 3 and 4 illustrate the two main limitations of single-pulse ISRS, i.e., small vibrational amplitude and lack of mode selectivity. Multiple-pulse excitation (multiple-pulse ISRS) can be used to help overcome these limitations. We demonstrate this point by using multiplepulse ISRS to drive the $80-\mathrm{cm}^{-1}$ librational mode (an in-phase libration of the molecule pairs) and the 104-cm translational mode selectively and individually in $\alpha$ perylene crystal. Figure 5(A) shows the intensity profile of a $2.39-\mathrm{THz}\left(79.6-\mathrm{cm}^{-1}\right)$ excitation-pulse train, timed to match the vibrational period of the $80-\mathrm{cm}^{-1}$ mode, and Fig. 5(B) shows the resulting ISRS data recorded from $\alpha$-perylene with all pulses polarized parallel to the $b$ crystallographic axis. Initially the signal is dominated by the crystal's electronic responses to each of the pulses in the sequence. However, the vibrational signal builds up as the pulse train progresses. After approximately 10 pulses, the ISRS signal that is due to the amplified $80 \mathrm{~cm}^{-1}$ mode becomes apparent. By the end of the inputpulse train $(t \sim 3 \mathrm{ps})$, the ISRS signal is approximately as intense as the strongest electronic scattering peak. Furthermore, the ISRS signal after the pulse train has ended appears to be oscillatory at a single frequency that is equal

(C)

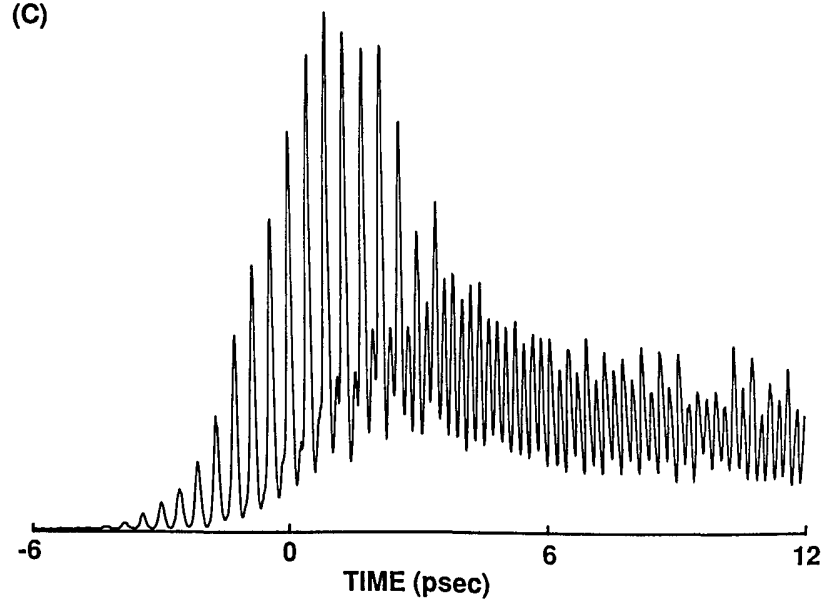

(D)

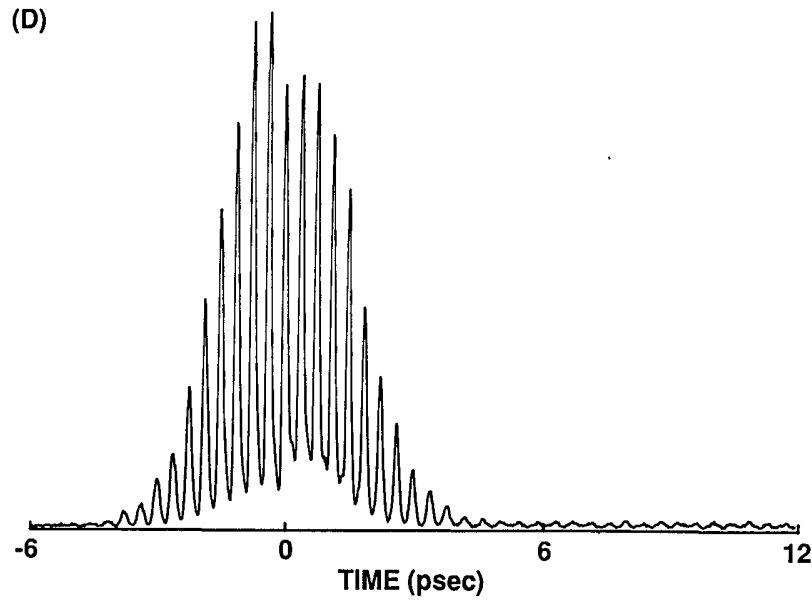

Fig. 5. Multiple-pulse ISRS data recorded from $\alpha$-perylene with $T<10 \mathrm{~K}$ and with all pulses linearly polarized parallel to the $b$ crystallographic axis. (A) Intensity cross-correlation measurement of $2.39-\mathrm{THz}\left(79.6-\mathrm{cm}^{-1}\right)$ pulse train used for resonant excitation of the $\alpha$-perylene's $80-\mathrm{cm}^{-1}$ librational mode. ISRS data (B) for 79.6 $\mathrm{cm}^{-1}$ excitation, (C) for 77.8- $\mathrm{cm}^{-1}$ excitation, (D) for $88.7-\mathrm{cm}^{-1}$ excitation. 


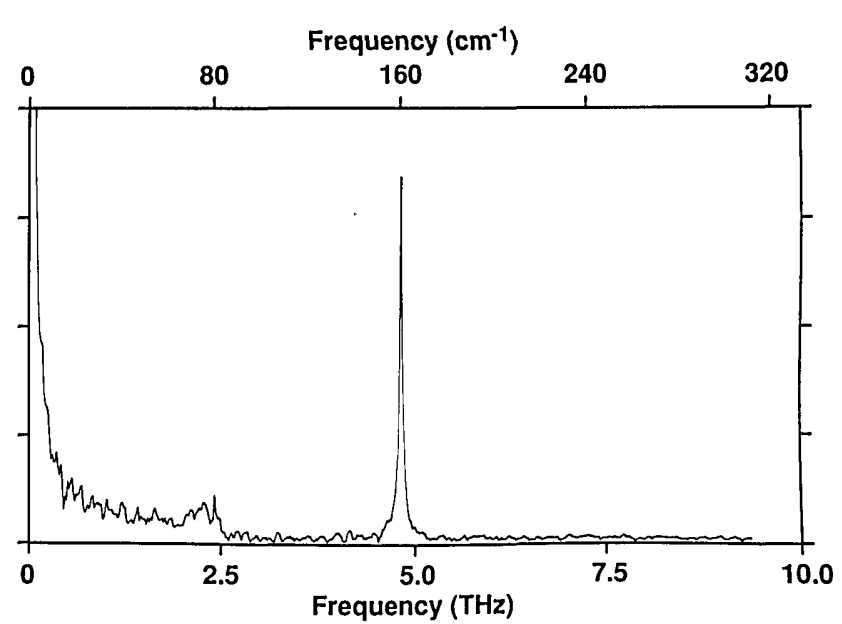

Fig. 6. Fourier transform of ISRS data, corresponding to resonant excitation of the $80-\mathrm{cm}^{-1}$ librational mode, as in Fig. 5(B).

to twice the vibrational frequency. ${ }^{2,8,15}$ This observation is confirmed by Fourier analysis of the data, as shown in Fig. 6. A single sharp line appears at $160 \mathrm{~cm}^{-1}(4.8 \mathrm{THz})$. Similar data are obtained with the polarizations parallel to the $a$ crystallographic axis, although both electronic and vibrational signals are reduced by approximately a factor of 3 compared with those for the case of $b$ polarization. Clearly, these data demonstrate considerable modeselective vibrational amplification.

We also performed multiple-pulse ISRS experiments with the repetition rate of the input-pulse train detuned slightly from the $80-\mathrm{cm}^{-1}$ librational period. Examples of our data are shown in Figs. 5(C) and 5(D). As the detuning is increased, the strength of the phonon response gradually decreases, but the oscillations remain monochromatic with the same $160-\mathrm{cm}^{-1}$ frequency. In Fig. $5(\mathrm{C})$ the excitation repetition rate is changed to $2.33 \mathrm{THz}\left(77.8 \mathrm{~cm}^{-1}\right)$, corresponding to a relatively small detuning of $0.06 \mathrm{THz}$. Vibrational amplification is still achieved, although the strength of the vibrational signal is reduced by approximately a factor of 2 compared with that for resonant excitation. For substantially larger detuning [Fig. 5(D), $2.66-\mathrm{THz}$ excitation] the vibrational signal is strongly suppressed, although instantaneous electronic scattering peaks remain. These observations are consistent with a simple picture of a forced harmonic oscillator. The response of the oscillator depends on the overlap of the excitation power spectrum with the natural frequency of the oscillator. The finite duration of our excitation-pulse train ( $\sim 4.8 \mathrm{ps}$ FWHM) leads to an excitation linewidth of $\sim 0.09 \mathrm{THz}\left(3.1 \mathrm{~cm}^{-1}\right)$ FWHM. Thus, for the data of Fig. 5(C), the detuning is comparable with the half-width of the excitation spectrum, in accord with the 50\% reduction of the phonon response. We may equivalently consider the requirement that the driving pulse train must remain in phase with the natural vibrational frequency (within $\pi$ radians) in order for vibrational amplification to occur. For the detuning of $0.06 \mathrm{THz}$ in Fig. 5(C) a phase difference of $0.58 \pi$ accumulates during a 4.8-ps time interval, equal to the excitation FWHM; therefore amplification can be achieved, though with some decrease in efficiency. The 0.27-THz detuning in Fig. 5(D) corresponds to an accumulated phase difference of $\sim 2.5 \pi$, and therefore vibrational amplification is not observed. For detunings such that amplification can be achieved, the vibrational signal remaining after the end of the driving pulse train is expected to reflect the natural response of the harmonic oscillator. This is exactly what is observed: the vibrational signal oscillates at the same $160-\mathrm{cm}^{-1}$ frequency, independent of the excitation frequency.

Because multiple-pulse ISRS results in selective amplification of a single phonon mode, the phonon decay dynamics can easily be determined. Figure 7(A) shows scattering data for resonant excitation of the $80-\mathrm{cm}^{-1}$ mode plotted over a sufficient time interval that the phonon decay is evident. The coherent oscillations decay with a $1 / e$ time of $14.3 \mathrm{ps}$, indicating a phonon dephasing time of $28.6 \mathrm{ps}$ at $10 \mathrm{~K}$. Note that the oscillations in the signal occur at twice the vibrational frequency and decay at twice the decay rate because the diffraction intensity is proportional to the square of the dielectric response (and therefore to the square of the vibrational displacement) in the medium. Figures 7(B)-7(E) show multiple-pulse ISRS data taken at several different temperatures. As the temperature is increased the phonon dephasing time $T_{2}$ decreases. From our data we estimate that $T_{2}=19 \mathrm{ps}$ at $20 \mathrm{~K}, 7$ ps at $50 \mathrm{~K}$, and $\sim 3.8 \mathrm{ps}$ at $100 \mathrm{~K}$, in reasonable agreement with estimates previously obtained from single-pulse ISRS data. ${ }^{15}$ We note that when the phonon dephasing time becomes comparable with or shorter than the duration of the driving pulse train, the vibrational amplification becomes less effective, and the phonon response becomes harder to distinguish from the electronic responses. At room temperature $T_{2}$ is of the order of $1 \mathrm{ps},{ }^{15}$ and the vibrational signal persists only during the trailing portion of the exciting pulse train. These data point to one fundamental constraint governing multiplepulse ISRS, namely, that the number of pulses that can be effectively used for vibrational amplification is limited by the phonon dephasing time. Increasing the duration of the pump-pulse train beyond the dephasing times of Ramanactive modes leads neither to increased vibrational amplification nor to increased mode selectivity.

In addition to the $80-\mathrm{cm}^{-1}$ mode, we use multiple-pulse ISRS to drive the 104- $\mathrm{cm}^{-1}$ mode, whose motion is essentially a vibration of a pair of neighboring perylene molecules against each other. Examples of data taken at low temperature are plotted in Fig. 8. For pulses polarized along the $a$ crystallographic axis, the degree of vibrational amplification achieved for the 104- $\mathrm{cm}^{-1}$ mode is comparable with that for the $80-\mathrm{cm}^{-1}$ mode (although the intensity of the 104- $\mathrm{cm}^{-1}$ signal relative to the maximum $80-\mathrm{cm}^{-1}$ signal is reduced by approximately a factor of 6 , indicating a smaller differential polarizability for this mode). As with the $80-\mathrm{cm}^{-1}$ mode, a slight detuning of the multiple-pulse timing led to a vibrational response with the same frequency and dephasing rate but of weaker amplitude. For pulses polarized along the $b$ crystallographic axis, the vibrational signal appears to be quite weak. The difference in appearance between Figs. 8(A) and $8(B)$ arises because the ISRS signal from the $104-\mathrm{cm}^{-1}$ mode is two times lower for $b$-polarized pulses than for $a$-polarized pulses, whereas the electronic scattering signal is three times higher for $b$ polarization.

We carried out numerical simulations to model our experimental results. Our modeling is an adaptation of an analysis that was previously developed for single- 

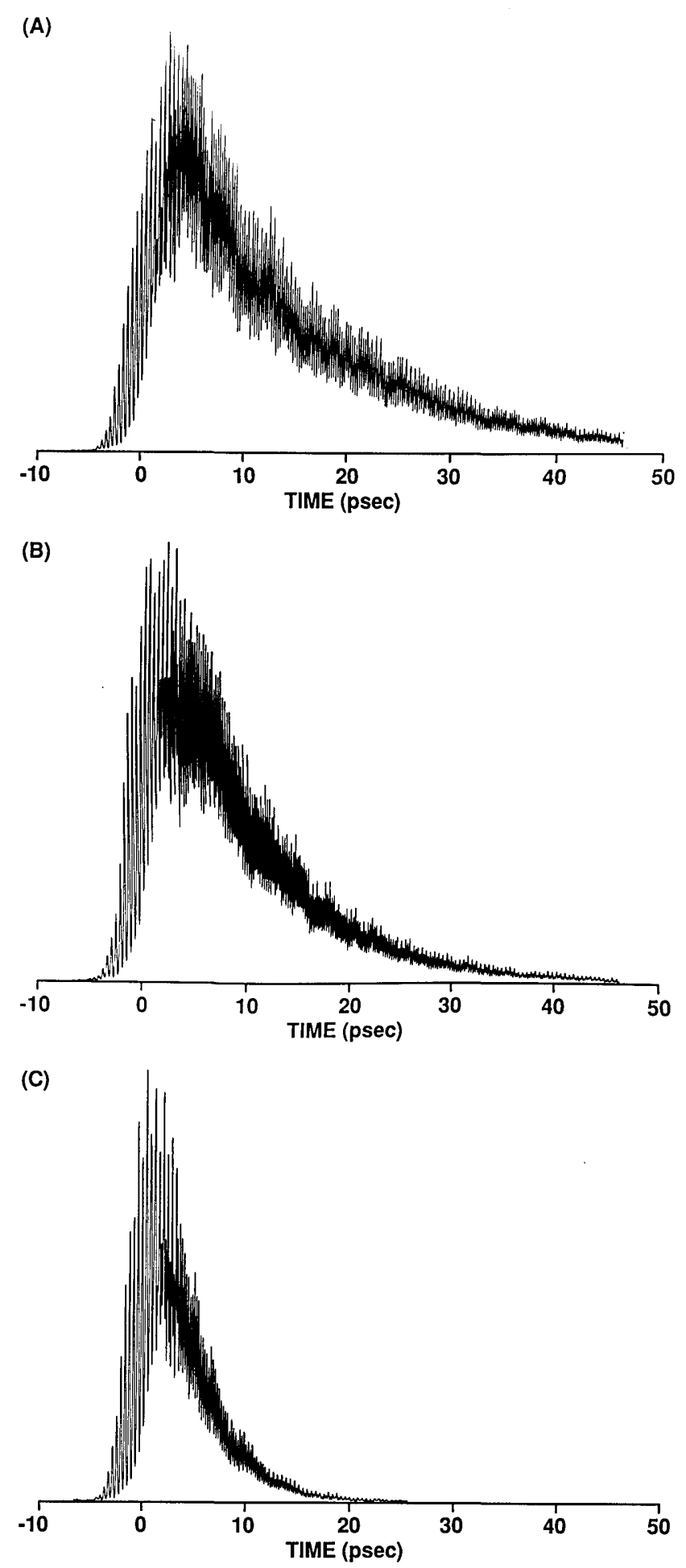

pulse ISRS experiments and that was described in detail elsewhere. ${ }^{20}$ We treat the individual vibrational modes as classical harmonic oscillators. Recently a quantummechanical analysis of multiple-pulse ISRS excitation was presented. ${ }^{21}$ In Appendix A we discuss the relation between the classical and the quantum-mechanical interpretations of ISRS excitation.

We begin our analysis of multiple-pulse ISRS by describing the intensity profile $I(x, t)$ of the shaped pump-pulse train that is incident upon the sample, using the following approximate form:
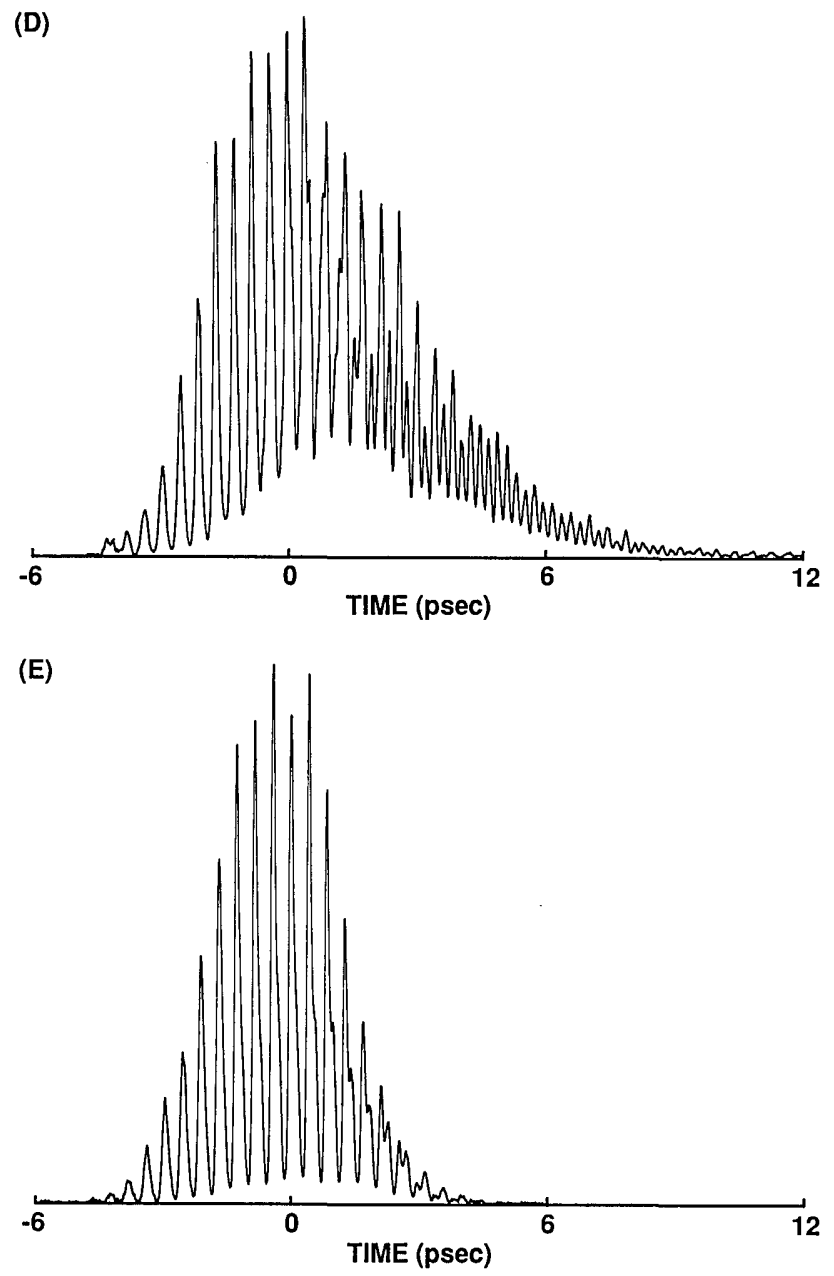

Fig. 7. Multiple-pulse ISRS data, showing decay of the $80-\mathrm{cm}^{-1}$ mode at various temperatures. Note that a shorter time scale is used for plots (D) and (E). (A) $T=10 \mathrm{~K}$, (B) $T=20 \mathrm{~K}$, (C) $T=$ $50 \mathrm{~K}$, (D) $T=100 \mathrm{~K}$, (E) $T=295 \mathrm{~K}$.

$$
I(x, t)=\sum_{n} I_{o} \exp \left[\frac{-(t-n T)^{2}}{t_{p}^{2}}-\frac{n^{2}}{N_{p}^{2}}\right] \cos ^{2} k x .
$$

Here $T$ is the spacing between the pulses, $t_{p}$ is a measure of the pulse duration of the individual pulses in the sequence, $N_{p}$ is a measure of the number of pulses within the pulse train, and $I_{o}$ is the peak intensity. The index $n$ takes on both positive and negative values. The first term in the exponential gives the temporal profile of the individual pulses (assumed to be Gaussian), and the second term describes the temporal profile of the pulse train (also 

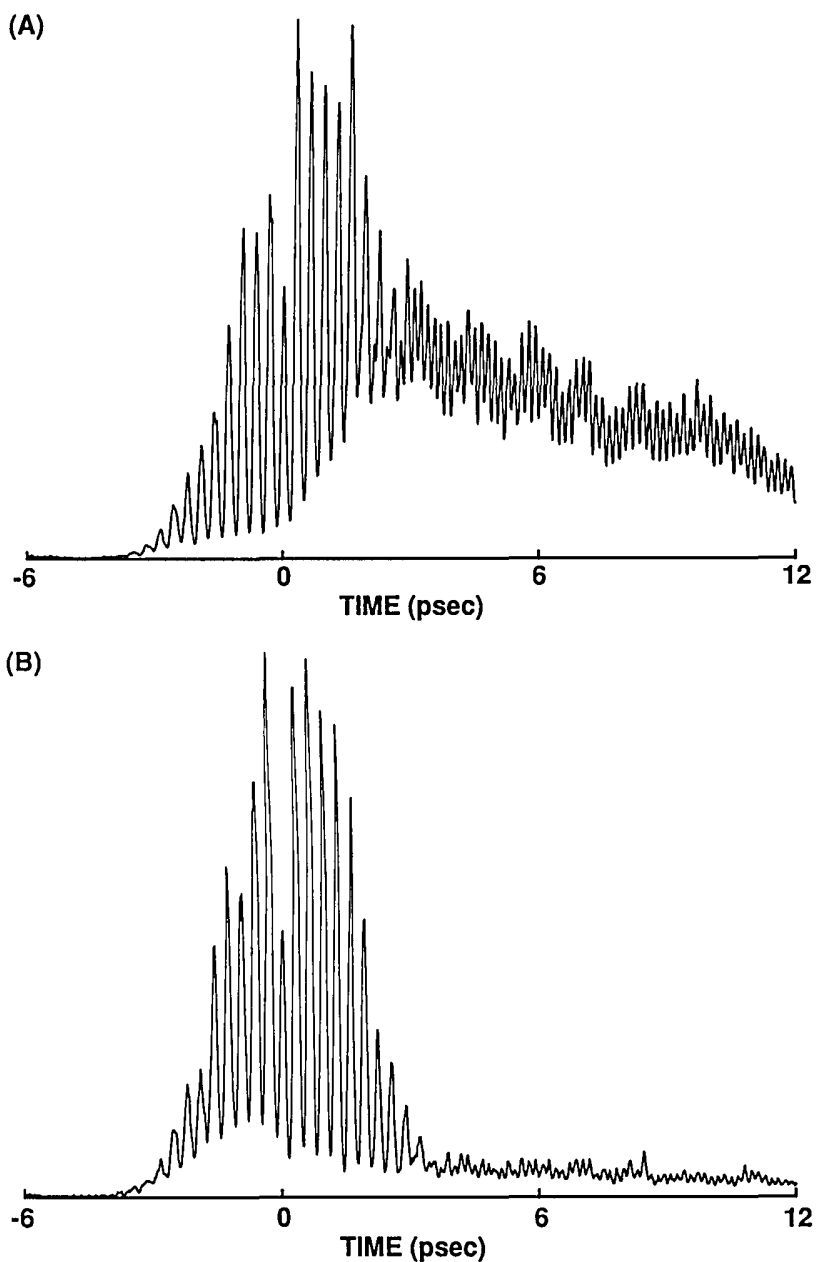

Fig. 8. ISRS data for resonant multiple-pulse excitation of the $104-\mathrm{cm}^{-1}$ vibrational mode in $\alpha$-perylene. The temperature is $T=10 \mathrm{~K}$. (A) All pulses polarized along the $a$ crystallographic axis. (B) Polarization along the $b$ axis.

assumed to be Gaussian). The $\cos ^{2} k x$ term describes the spatial interference of the two crossed pump beams. The vibrational response of the sample that is due to the $i$ th vibrational mode is characterized by an impulse response function (Green's function) $G_{i}(t)$, given by

$$
G_{i}(t)=C_{i} \exp \left(-\gamma_{i} t\right) \sin \omega_{i} t
$$

where $\gamma_{i}, \omega_{i}$, and $C_{i}$ are, respectively, the damping rate, the natural vibrational frequency, and the relative strength of the $i$ th mode. The convolution of $I(x, t)$ and $G_{i}(t)$ gives the time- and space-dependent vibrational response, $Q_{i}(x, t)$, that is due to the $i$ th mode, i.e.,

$$
Q_{i}(x, t)=\int_{-\infty}^{t} \mathrm{~d} t^{\prime} I\left(t^{\prime}\right) G_{i}\left(t-t^{\prime}\right)
$$

The change in the dielectric constant, $\delta \epsilon(x, t)$, is given by

$$
\delta \epsilon(x, t)=\sum_{i} \frac{\partial \epsilon}{\partial Q_{i}} Q_{i}+2 n_{0} \gamma I(x, t),
$$

where the summation is carried out over all the modes of the crystal. The term $2 n_{0} \gamma I(x, t)$ describes the essentially instantaneous index change that is due to the bound electron nonlinearity of the material. $\gamma$ is the usual nonlin- ear index coefficient in square centimeters per watt, and $n_{0}$ is the low-intensity refractive index. The ISRS diffraction efficiency is proportional to the square of the dielectric response at the grating peaks, i.e., to $\delta \epsilon^{2}(x=0, t)$. The actual ISRS signal is taken to be the convolution of $\delta \epsilon^{2}(x=0, t)$ over the probe-pulse intensity profile (assumed to be identical to the profile of the individual pulses in the pump-pulse sequence).

In Fig. 9 we show simulation results for multiple-pulse excitation of the $80-\mathrm{cm}^{-1}$ mode of $\alpha$-perylene. For these simulations we set $C_{i}$ equal to zero for modes other than the $80-\mathrm{cm}^{-1}$ mode. Our omission of the contributions of the other vibrational modes is justified because of the strong mode selectivity of the multiple-pulse ISRS technique, as evidenced by the excellent agreement between data and simulation. For Fig. 9(A) the excitation-pulse repetition rate exactly matches the $80-\mathrm{cm}^{-1}$ natural vibrational frequency, and the numerical results are in close agreement with the experimental data [Fig. 5(B)]. For the parameters in Eq. (1) we used $t_{p}=42 \mathrm{fs}, N=7$, and $T=419 \mathrm{fs}$, in accordance with our measurement of the excitation-pulse train shown in Fig. 5(A). In addition, we

(A)

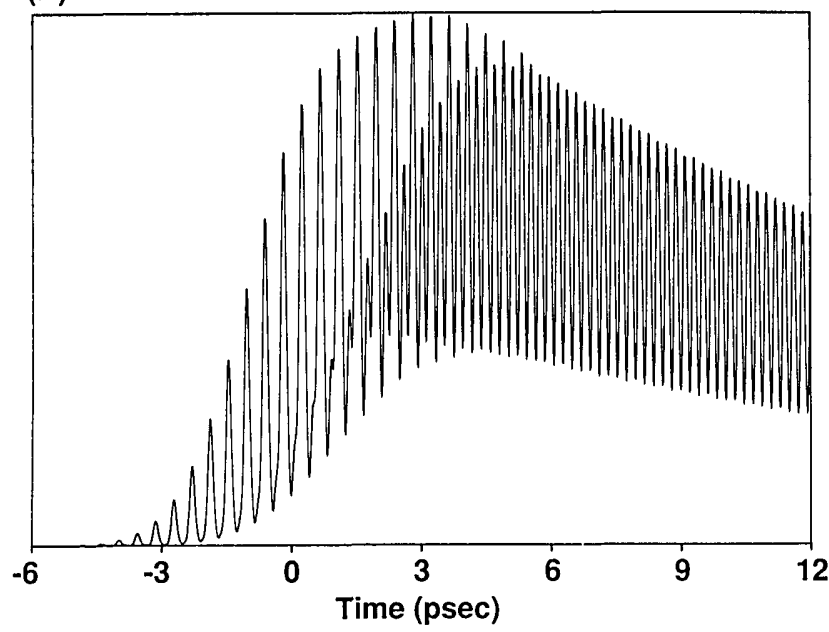

(B)

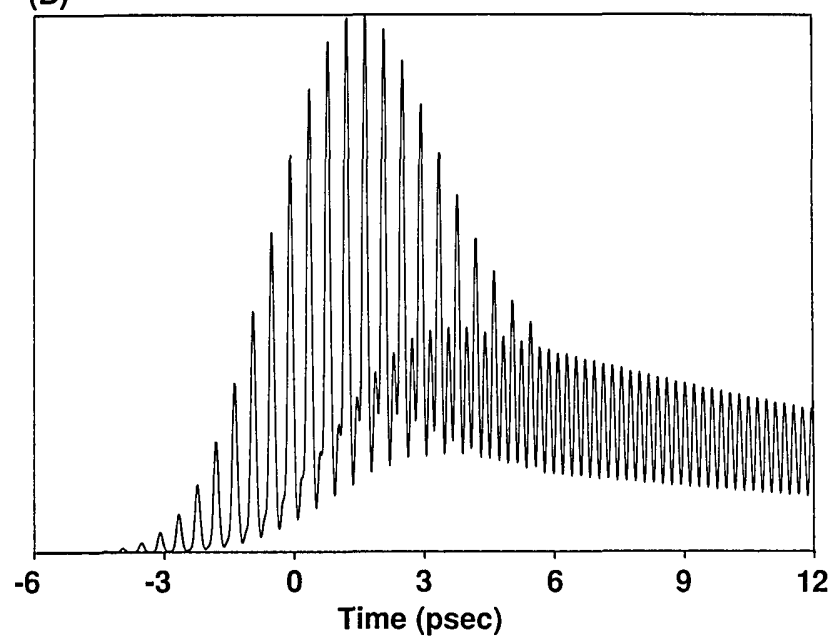

Fig. 9. Simulation results for multiple-pulse excitation of the 80 $\mathrm{cm}^{-1}$ mode in $\alpha$-perylene. (A) On resonance, $2.39-\mathrm{THz}\left(79.6-\mathrm{cm}^{-1}\right)$ pulse repetition rate. (B) Detuned from resonance, $2.33-\mathrm{THz}$ $\left(77.8-\mathrm{cm}^{-1}\right)$ pulse repetition rate. 


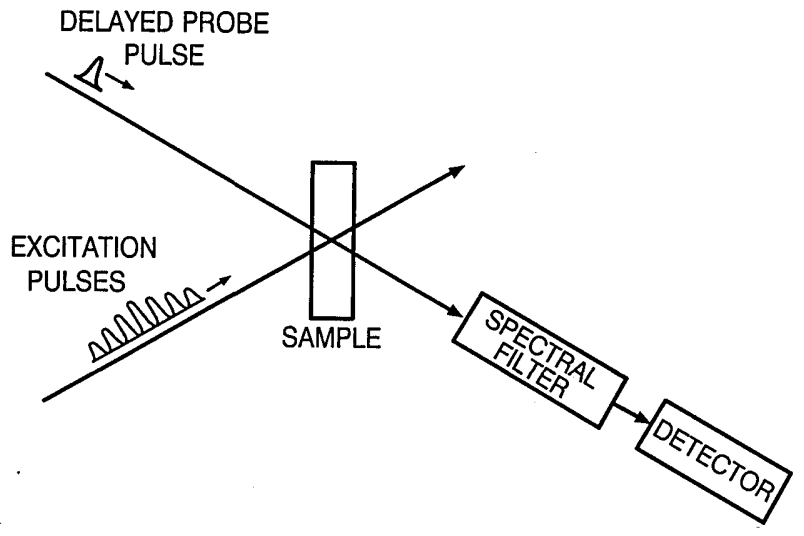

Fig. 10. Experimental arrangement for forward (or pump-probe) ISRS experiments with multiple-pulse excitation.

used $\gamma^{-1}=28.6 \mathrm{ps}^{-1}$ for the $80-\mathrm{cm}^{-1}$ mode, and we adjusted the relative strength of the vibrational and electronic nonlinearities to give the best fit with the data. Numerical simulations were also performed for excitation-pulse repetition rates detuned from resonance. Figure $9(B)$ shows the simulation results for a $2.33-\mathrm{THz}$ pulse repetition rate, corresponding to $T=429$ fs (all the other parameters were identical to those used for the resonant excitation simulation). Again the simulation is in close agreement with the data [Fig. 5(C)].

\section{PUMP-PROBE EXPERIMENTS}

In addition to the transient-grating experiments reported in the sections above, we also performed forward ISRS (or pump-probe) experiments using multiple-pulse excitation. The experimental geometry is sketched in Fig. 10. The single pump beam, consisting of an appropriately timed terahertz-rate sequence of femtosecond pulses, resonantly drives a desired phonon mode in the Raman-active sample. Depending on its delay, the probe pulse can arrive at the sample either in or out of phase with the excitationpulse train and so can either amplify or oppose, respectively, the coherent vibrational motion. When the probe is timed to amplify the motion, it gives up energy to the vibrational mode and emerges from the sample Doppler shifted to the red. Conversely, when the probe is timed to oppose the coherent vibrational motion, it removes energy from the sample and emerges Doppler shifted to the blue. ${ }^{8}$ We emphasize that, unlike conventional pumpprobe measurements in which the pump modifies the material absorption seen by the probe, our experiments are performed with wavelengths for which the sample is nonabsorbing. The change in the probe frequency induced by the pump actually results from the time-varying refractive index $\delta n(t)$ accompanying the coherent vibrational motion, which can shift the probe to either longer or shorter wavelengths, depending on the sign of $\mathrm{d} \delta n / \mathrm{d} t$. We detect this spectral shift by passing the probe through a bandpass filter placed after the sample. With the filter passband set to the blue extremum of the spectrum, for example, a shift to the blue (red) translates into an increase (decrease) in the average transmitted probe power. The experiment then consists of measuring the probe power transmitted through the filter as a function of the relative probe delay. Similar forward ISRS experiments were reported for single-pulse excitation. ${ }^{2}$ The current study constitutes the first report to our knowledge of forward ISRS with multiple-pulse excitation.

We demonstrated this technique by detecting spectral shifts induced by $\alpha$-perylene's $80-\mathrm{cm}^{-1}$ librational mode. Except for the difference in the optical arrangement, the experimental setup is similar to that used in the transientgrating measurements. The pump beam is a $2.39-\mathrm{THz}$ pulse train, as in Fig. 5(A), which resonantly drives the $80-\mathrm{cm}^{-1}$ mode, and both the pump and the nearly collinear probe beams are polarized along the $b$ crystallographic axis. The crystal is maintained at low temperature $(\sim 10 \mathrm{~K})$. The optical bandpass filter is essentially identical to our optical pulse-shaping apparatus. A grating and a lens are used for spatial dispersion of the optical frequencies contained within the probe pulse, and an iris placed one focal distance away from the lens is used to select the desired portion of the spectrum. A second lens reassembles the remaining frequency components into a single spatial beam, which is incident upon a single detector. When the iris is removed, the detector measures just the total power of the probe beam. Thus this setup provides an easily adjustable bandpass filter. ${ }^{22}$

Examples of our data are shown in Fig. 11. For Figs. 11(A) and 11(B), the iris is positioned to pass only the shortest-wavelength portion or the longest-wavelength portion of the spectrum, respectively. In each case the opening of the iris is adjusted so that it transmits one third of the total power in the probe. Both curves show a well-defined series of oscillations that build up during the excitation-pulse train. The oscillation is monochromatic with a $2.40-\mathrm{THz}\left(80 \mathrm{~cm}^{-1}\right)$ frequency, in contrast to the earlier transient-grating data, which showed oscillations at twice the phonon frequency $\left(160 \mathrm{~cm}^{-1}\right)$. This difference is expected, since the pump-probe experiment measures a signal that is directly proportional to the vibrationally induced index change (more precisely, to the time derivative of the index change), while the transient-grating experiment measures a signal that is proportional to the square of the index change. For time delays such that the probe arrives after the end of the excitation-pulse train $(t>4 \mathrm{ps})$, the oscillations in the two curves are essentially equal in amplitude but precisely out of phase, confirming that the signal corresponds to a periodic shifting of the probe spectrum alternately to the red and to the blue. From the magnitude of the pump-probe signal ( $-1 \%$ transmission change), we estimate that the spectral shift amounts to $\sim 0.1 \mathrm{~nm}(\sim 1 \%$ of the probe bandwidth).

We note that the portions of Figs. 11(A) and 11(B) for which the probe overlaps the pump sequence $(t<4 \mathrm{ps})$ are quite different, and this suggests that there is an additional contribution to the pump-probe signal during this time. Figure 11(C) shows pump-probe data taken with the iris removed from the bandpass filter, so that the signal reflects changes in the transmitted probe energy but is insensitive to spectral shifts. The data show a series of sharp dips in the probe transmission, with the same time signature as the excitation pulse sequence. We attribute these dips to two-photon absorption (TPA), occurring when the probe coincides with individual pump pulses. Thus we surmise that the data in Figs. 11(A) and 11(B) consist of contributions from both TPA and ISRS spectral shifts. We separate out these distinct contributions by noting 
(A)

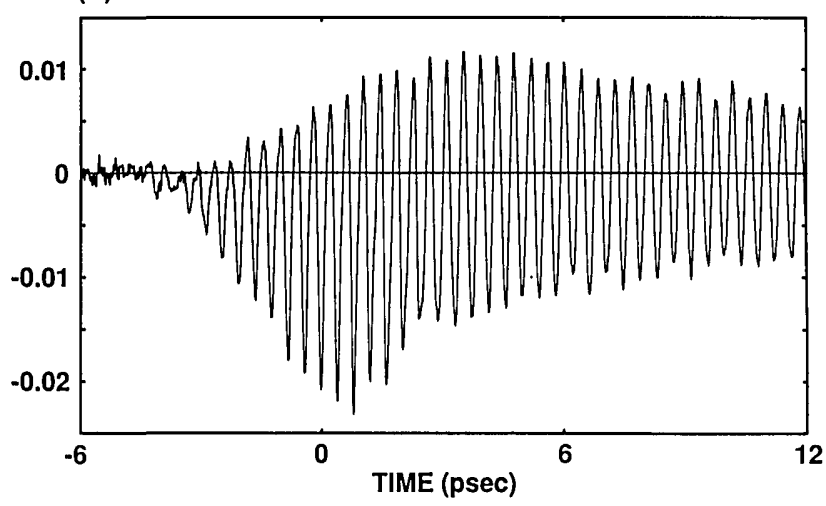

(B)

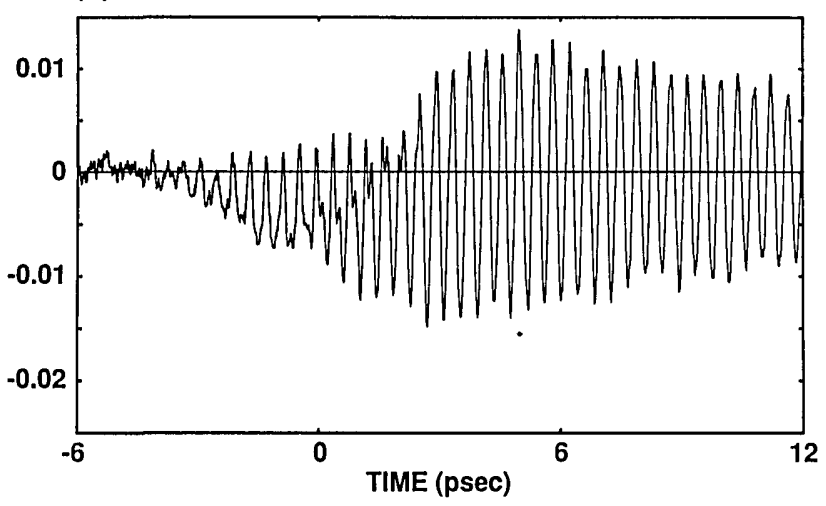

(C)

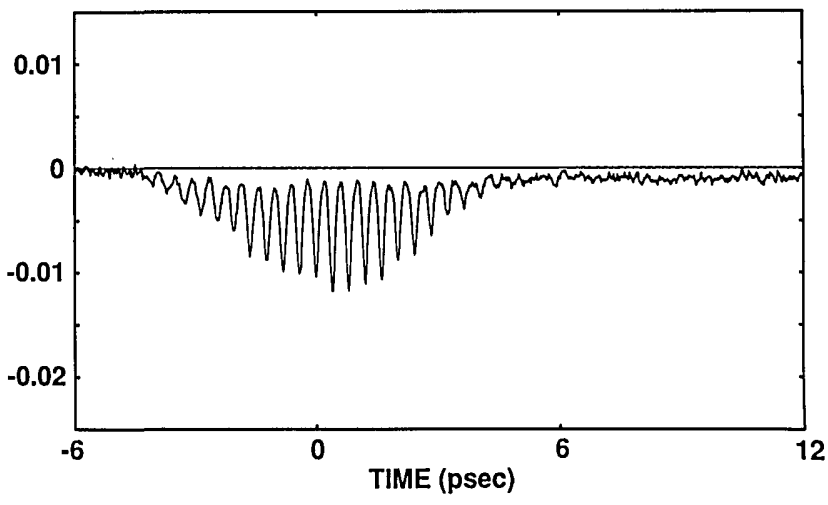

that the TPA signal should be identical and of the same polarity for both curves, whereas the ISRS signal is of opposite polarity from one curve to the other. By looking at the sum of the curves in Figs. 11(A) and 11(B), we obtain the TPA contribution, plotted in Fig. 11(D), which is essentially identical to the curve of Fig. 11(C). By looking at the difference of the curves of Figs. 11(A) and 11(B), we eliminate the TPA contribution and isolate the oscillatory ISRS signal. The result, Fig. 11(E), shows quite clearly that the ISRS oscillations observed in the pump-probe geometry build up throughout the pump-pulse sequence in much the same way as do oscillations observed in the transient-grating geometry.

It is now of interest to comment on the timing relationship between the ISRS oscillations and the coherent vibrational motion induced in the sample by the multiple-pulse excitation. As discussed elsewhere, ${ }^{8}$ each excitation pulse
(D)

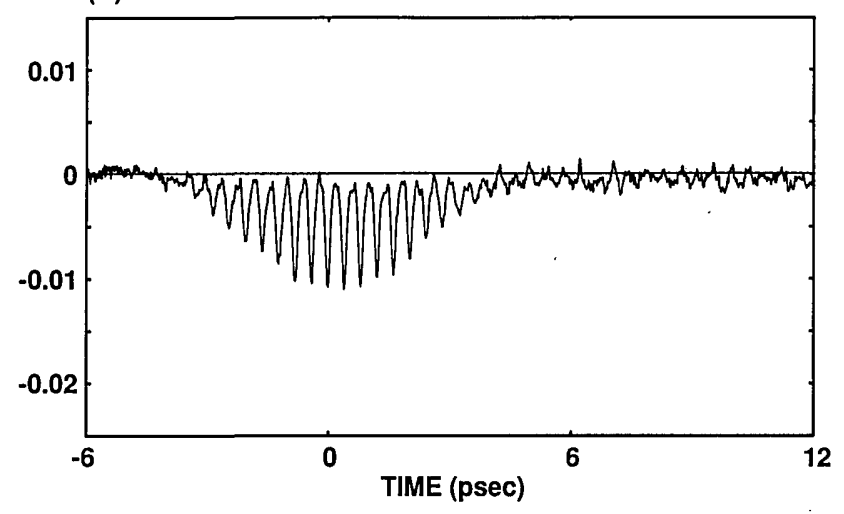

(E)

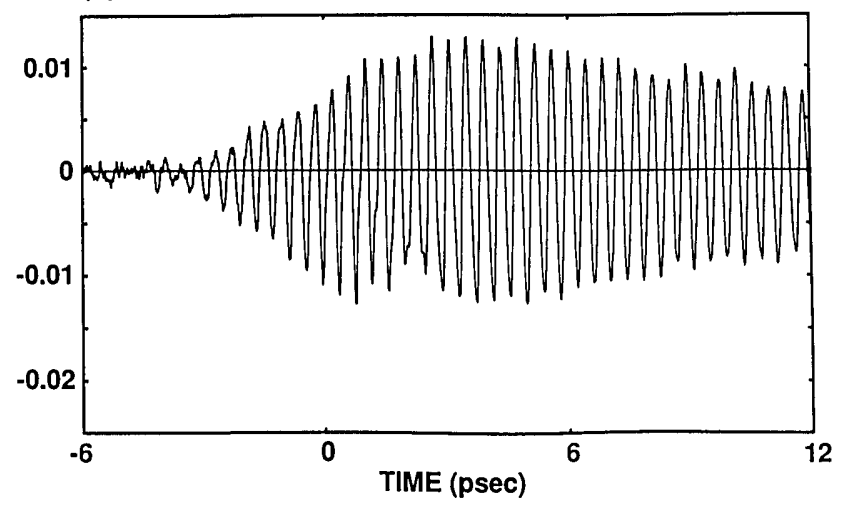

Fig. 11. Pump-probe ISRS measurements of $\alpha$-perylene at $T=$ $10 \mathrm{~K}$, in conjunction with resonant multiple-pulse excitation of the $80-\mathrm{cm}^{-1}$ librational mode. (A) Shortest-wavelength portion of the probe detected. (B) Longest-wavelength portion detected. (C) Entire probe spectrum detected. (D) Sum of (A) and (B).

(E) Difference of (A) and (B).

exerts an impulse force, which pushes the material system along its vibrational coordinates in the direction leading to a positive refractive-index change. For resonant multiple-pulse excitation, the material will have maximum vibrational velocity and minimum vibrational displacement at the time of the exciting pulses. Furthermore, because $\mathrm{d} \delta n / \mathrm{d} t>0$ at the time of the exciting pulses, the probe pulse will experience a maximum red shift when it arrives in phase with the pump-pulse train and a maximum blue shift when it arrives exactly out of phase with the pumppulse train. This is exactly the timing relationship indicated by the data in Fig. 11.

The timing relationship is somewhat different for transient-grating ISRS experiments, which are sensitive to the refractive-index change $\delta n$, not to its time derivative. Maximum diffraction occurs when the probe arrives at times of maximum vibrational displacement (and hence 
minimum vibrational velocity). Therefore ISRS oscillations measured in the transient-grating geometry should be in quadrature with oscillations measured in the pumpprobe geometry. Figure 12 shows grating and pump-probe data plotted together over the same 1-ps time interval. As expected, extrema in the pump-probe data correspond to minima in the diffraction data, and maxima in the diffraction data correspond to zero crossings of the pumpprobe data.

\section{DISCUSSION}

At this point we outline several limitations of the multiplepulse ISRS technique. First, ISRS requires that the individual excitation pulses be significantly shorter than one vibrational period, and this sets an upper limit on accessible vibrational frequencies. Similarly, in order to generate bursts of clean, well-isolated pulses it is necessary that the pulse repetition period should be at least twice the pulse width. To date, using 75-fs input pulses, we have generated bursts of pulses at repetition rates of up to $6 \mathrm{THz}$, and therefore vibrations at frequencies of up to $200 \mathrm{~cm}^{-1}$ should be accessible. By using pulse-shaping techniques in conjunction with shorter pulses, it should be possible for one to generate pulse trains to drive vibrations in the $1000-\mathrm{cm}^{-1}$ range.

A second limitation is imposed by the dephasing rate of the vibrational mode of interest. As mentioned in Section 4, modes that are damped after just a few oscillations can be driven by only a small number of pulses and therefore cannot be amplified so much as lightly damped modes can be. Both pure dephasing and the inhomogeneous dephasing that is associated with local variations in condensed-phase structure, leading to local shifts of the vibrational frequency, can contribute to damping, which will limit multiple-pulse ISRS. ${ }^{23}$ In general, one can take the best advantage of multiple-pulse ISRS excitation by increasing the number of pulses in the pulse train until the duration of the pulse train at least matches the phonon
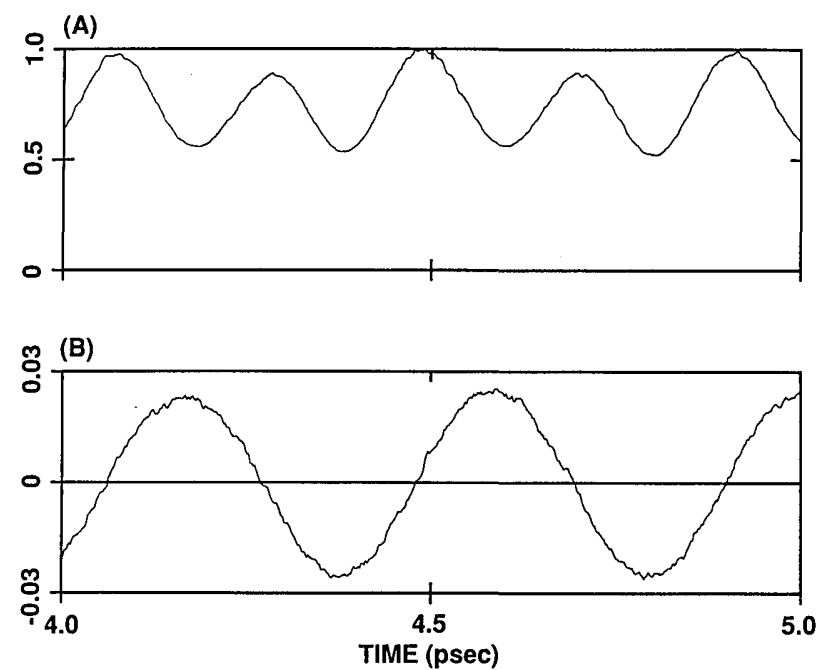

Fig. 12. Comparison of (A) transient-grating ISRS data and (B) pump-probe ISRS data, both corresponding to resonant excitation of the $80-\mathrm{cm}^{-1}$ librational mode at $T=10 \mathrm{~K}$. The extrema in the pump-probe data correspond to minima in the diffraction data, and the maxima in the diffraction data correspond to zero crossings of the pump-probe data. dephasing time. Additional pulses will not substantially increase the amplitude of the selected mode, although they may act to suppress further other modes with longer dephasing times.

To the extent that large vibrational amplitudes are desired, the main limitations are unwanted optical responses, including those that lead to sample damage of various forms. In fact, coherent vibrational amplitudes in the angstrom range could in principle be driven by single femtosecond pulses, were it not for multiphoton absorption, continuum generation, sample damage, and so on, which occur at the extremely high peak intensities required. Splitting up a single pulse into a sequence of less intense pulses is one way of channeling the light energy into ISRS excitation and not into unwanted processes. For single-pulse ISRS, the induced vibrational amplitude is proportional to the excitation-pulse energy. For resonant multiple-pulse ISRS, the final vibrational amplitude (neglecting dephasing) is proportional to the total energy in the entire pulse train. Therefore the ability to drive larger vibrational amplitudes by multiple-pulse ISRS will depend on the extent to which multiple-pulse excitation makes possible increased excitation energies.

In the current experiments with $\alpha$-perylene, the coherent vibrational oscillations achieved are still in the smallamplitude limit. By using formulas given in Refs. 8 and 15 , we can determine the amount of vibrational motion from the measured diffraction efficiency and the known experimental parameters. For the $104-\mathrm{cm}^{-1}$ phonon mode, which corresponds to vibration of neighboring, planar perylene molecules against one another, the peak diffraction efficiency for multiple-pulse excitation is $\sim 0.02 \%$, and the estimated peak-to-peak change in molecular separation is of the order of $10^{-4} \AA$, compared with an equilibrium molecular separation of $0.2 \AA$. For the $80-\mathrm{cm}^{-1}$ librational mode, in which the molecule pairs are librating in phase, the diffraction efficiency is $\sim 0.12 \%$, and the estimated peak-to-peak angular deviation is $0.001^{\circ}$. The magnitude of the induced vibrational motion is limited by sample damage, which occurs at total fluences of $\sim 3$ and $5 \mathrm{~mJ} / \mathrm{cm}^{2}$ for single- and multiple-pulse excitation, respectively. Because of the difference in damage threshold for multiple- versus single-pulse excitation, one expects that a $60 \%$ greater vibrational amplitude could be achieved with multiple-pulse excitation; this prediction is in accord with the highest diffraction efficiency observed for multiple-pulse relative to single-pulse excitation. Because linear absorption is quite weak in $\alpha$-perylene at $620 \mathrm{~nm}$, we initially expected that sample damage would be linked to two-photon absorption and that substantially higher energies would be possible with multiple-pulse excitation, for which the peak intensities would be much lower. The similarity of the damage thresholds for singleand for multiple-pulse excitation indicates that, in this crystal, damage from sequential processes, such as multistep absorption, may play an important role in limiting the total energy that can safely be used.

There are many materials with damage thresholds that are substantially higher than that of the organic molecular crystal $\alpha$-perylene, and in such materials it might be possible to induce correspondingly stronger vibrational amplitudes through ISRS. Silicon, for example, has a damage threshold of the order of $100 \mathrm{~mJ} / \mathrm{cm}^{2}$ for femto- 
second above-band-gap excitation. Materials that combine a high damage threshold with a large Raman cross section would be especially favorable. Multiple-pulse ISRS will be particularly helpful in driving large-amplitude vibrational motion in cases in which unwanted nonlinear processes limit the optical intensity rather than the energy. This might occur in liquids and high-damage-threshold solids in which continuum generation or multiphoton absorption might constitute the limiting process.

Even with small vibrational amplitudes, multiple-pulse ISRS can be important for spectroscopy. As we have already seen, selective excitation of a single vibrational mode facilitates measurement of the phonon dephasing time. More important, ISRS excitation opens up the possibility of carrying out time-resolved spectroscopy of vibrationally distorted crystal lattices and molecules. ${ }^{8}$ For example, after ISRS excitation of optical phonons in III-V or II-VI semiconductors, one might be able to use femtosecond absorption spectroscopy to observe time-varying spectral shifts of the band gap or other electronic absorption features induced by the coherent vibrational motion. In these experiments the oscillation of the band gap would occur in synchronism with the phonon oscillations, and the maximum spectral shift would occur at instants of time corresponding to a maximum vibrational displacement. In order for one to interpret such experiments, it will be important to know the absolute timing of the vibrational response. As demonstrated in Section 5, multiplepulse ISRS makes possible the precise determination of the vibrational timing dynamics that will be required for these new types of spectroscopy.

\section{SUMMARY}

In summary, we have demonstrated multiple-pulse ISRS, an initial application of specially shaped sequences of femtosecond pulses to obtain improved optical control over molecular motion. We have used this technique to achieve mode-selective amplification of individual vibrational modes in $\alpha$-perylene molecular crystal. Substantially larger vibrational amplitudes should be attainable in materials with larger damage thresholds; through the use of multiple-pulse ISRS for precisely controlled excitation of desired phonon oscillations, time-resolved spectroscopy of vibrationally distorted molecules and crystal lattices should become possible. Furthermore, despite the power and flexibility that current pulse-shaping technology already provides, the technology is still evolving. For example, we recently demonstrated an electronically programmable pulse-shaping technique that is based on the use of a multielement liquid-crystal modulator in place of the prefabricated masks that are used in the current work. ${ }^{10}$ Other programmable pulse-shaping techniques are also being extended into the femtosecond regime. ${ }^{11}$ These developments could facilitate compensation for vibrational anharmonicity at large amplitudes, for example, by allowing the time between successive pulses in a sequence to be varied as necessary. In addition to ISRS, several other schemes proposed for optical control over molecular motion ${ }^{7}$ would also require specially designed femtosecond wave forms. It seems clear that femtosecond pulse shaping will play an increasingly important role in optical manipulation of molecular behavior.

\section{APPENDIX A： QUANTUM-MECHANICAL DESCRIPTION OF MULTIPLE-PULSE ISRS}

ISRS excitation with one pulse or a pulse sequence can, of course, be analyzed quantum mechanically as well as classically. A quantum-mechanical analysis of multiple-pulse ISRS excitation was presented recently. ${ }^{21}$ It is useful to consider the implications of this analysis and the limitations of the classical picture presented above.

For either one or many excitation pulses, the phasecoherent vibrational motion described classically in Section 4 can be expressed in terms of a coherent superposition of vibrational eigenstates of the form

$$
|\Psi(t)\rangle=\sum_{n=0}^{\infty} A_{n}|n\rangle,
$$

where $A_{n}$ specifies the amplitude and phase of the eigenstate $|n\rangle$. Immediately after excitation, all the $A_{n}$ 's are phase coherent. In the limit of a large driving force, $A_{n}$ is large for many $n$ up to high levels, and the classical and quantum-mechanical descriptions are equivalent. In this case the analogy between multiple-pulse ISRS and repetitive pushing of a child on a swing can be made in a general way. If the driving force is small, however, the coherent superposition of states, Eq. (A1), contains significant amplitude only in states $\left|A_{0}\right\rangle$ and $\left|A_{1}\right\rangle$, with $\left|A_{0}\right| \gg\left|A_{1}\right|$. In this case the coherent vibrational amplitude is proportional to $A_{1}$ and increases with multiple excitation pulses in a manner described classically by analogy to a child on a swing. Note that the amplitude can be related directly to an experimental observable (the intensity of coherent scattering, for example). The spectrum of amplitude eigenvalues is continuous, not discrete. The result of a measurement of coherent amplitude at a specified time can be predicted exactly, given knowledge of the wave function, Eq. (A1), and the measurement preserves the coherent superposition of states described by Eq. (A1).

A discussion of energy must be conducted more carefully, however. Like coherent amplitude, this quantity can be defined only in reference to an experimental observable. However, unlike for amplitude, measurement of energy yields one of a discrete spectrum of eigenvalues. The result of a particular measurement cannot be predicted exactly given knowledge of the wave function, Eq. (A1); only the probability of each result can be predicted. Finally, the measurement forces the system into an eigenstate, thereby destroying the coherent superposition of states. If $\left|A_{0}\right| \gg\left|A_{1}\right|$, then almost all the oscillators will end up in the $|0\rangle$ eigenstate after a measurement of energy (or after any dephasing process). Although multiple-pulse excitation does increase the coherent amplitude of each oscillator, it does not increase the energy of most oscillators (since measurement of energy after one or more excitation pulses still yields the lowest eigenvalue for most oscillators). Multiple excitation pulses increase the (small) probability that an oscillator will end up in the $|1\rangle$ state but do not increase the energy per oscillator for most oscillators. This is in contrast to the classical result.

As discussed above, under typical ISRS excitation conditions coherent vibrational displacements along translational optic phonon or molecular stretching coordinates are typically $\sim 10^{-4} \AA$. For independent oscillators, such as vibrational modes of molecules in a liquid or gas, this is 
far smaller than the quantum uncertainty of the lowest vibrational level and corresponds in quantum-mechanical terms to a coherent superposition of states with $\left|A_{0}\right| \gg$ $\left|A_{1}\right| \gg\left|A_{2}\right|$, i.e., the small-amplitude limit. ISRS excitation initiates coherent oscillations of all the irradiated molecules of the sample. After measurement of energy or other dephasing processes, the energy deposited by the excitation pulses is distributed among the localized molecular vibrations, with most molecules unexcited and with some having one quantum of vibrational energy.

For collective vibrations of a crystal lattice, the coherence length of the vibrational mode is an important factor. If this value is great (i.e., approaching macroscopic distance scales), then the zero-point quantum uncertainty in displacement along the phonon coordinate becomes extremely small, and ISRS excitation produces a coherent superposition of states with significant amplitudes for high-lying levels. After measurement of energy or other dephasing processes, the energy deposited by the excitation pulses is distributed among the independent oscillators in the irradiated volume. If the phonon coherence is long, there are few independent oscillators in this volume, each with many quanta of energy. If the coherence is short, then there are many independent oscillators in the irradiated volume, and each has either zero or one quantum of energy.

In a high-quality single crystal at low temperature, the coherence can be expected to be relatively long. It is likely although not certain that, in the case of $\alpha$-perylene below $10 \mathrm{~K}$, multiple-pulse ISRS excitation imparts more than one quantum of energy into the selected optic phonon mode.

Note that the classical analogy to a child on a swing is always valid for coherent vibrational amplitude. In general, we find it preferable to discuss the coherent superposition of states produced through ISRS excitation in terms of vibrational amplitude rather than energy. The result of a measurement of the former (but not the latter) quantity at any given time can be predicted exactly, given knowledge of the wave function, Eq. (A1). Similarly, when the system is in an eigenstate, it is usually more convenient to discuss energy for which measurement yields a predictable result.

We note finally that these considerations have no bearing on the prospects for achieving large vibrational amplitudes or driving chemical rearrangements through ISRS. Coherent large-amplitude oscillations correspond to a superposition of states with significant amplitudes for high-lying levels, at which point classical and quantummechanical descriptions become equivalent. This limit can be reached if and only if damage thresholds can be circumvented; the method of analysis will not influence the prospects.

\section{ACKNOWLEDGMENTS}

G. P. Wiederrecht and K. A. Nelson received support from National Science Foundation grant CHEM-8901722 and contributions from DuPont, Perkin-Elmer, and the Alfred P. Sloan Foundation.

\section{REFERENCES}

1. R. A. Mathies, C. H. Brito Cruz, W. T. Pollard, and C. V. Shank, Science 240, 777 (1988).

2. Y.-X. Yan, L.-T. Cheng, and K. A. Nelson, in Advances in Nonlinear Spectroscopy, R. J. H. Clark and R. E. Hester, eds., Vol. 16 of Advances in Spectroscopy Series (Wiley, Chichester, UK, 1988), pp. 299-355; S. Ruhman, A. G. Joly, B. Kohler, L. R. Williams, and K. A. Nelson, Rev. Phys. Appl. 22, 1717 (1987).

3. J. Chesnoy and A. Mokhtari, Phys. Rev. A 38, 3566 (1988).

4. F. W. Wise, M. J. Rosker, and C. L. Tang, J. Chem. Phys. 86, 2827 (1986).

5. M. Dantus, M. J. Rosker, and A. H. Zewail, J. Chem. Phys. 89, 6128 (1988); N. F. Scherer, C. Sipes, R. B. Bernstein, and A. H. Zewail, J. Chem. Phys. 92, 5239 (1990).

6. H. L. Fragnito, J.-Y. Bigot, P. C. Becker, and C. V. Shank, Chem. Phys. Lett. 160, 101 (1989).

7. D. Tannor, R. Kosloff, and S. A. Rice, J. Chem. Phys. 85, 5805 (1986); S. Shi, A. Woody, and H. Rabitz, J. Chem. Phys. 88, 6870 (1988).

8. Y.-X. Yan, E. B. Gamble, and K. A. Nelson, J. Chem. Phys. 83, 5391 (1985).

9. A. M. Weiner, J. P. Heritage, and E. M. Kirschner, J. Opt. Soc. Am. B 5, 1563 (1988).

10. A. M. Weiner, D. E. Leaird, J. S. Patel, and J. R. Wullert, Opt. Lett. 15, 326 (1990).

11. M. Haner and W. S. Warren, Appl. Phys. Lett. 52, 1458 (1988); W. S. Warren, Science 242, 878 (1988).

12. A. M. Weiner, D. E. Leaird, G. P. Wiederrecht, and K. A. Nelson, Science 247, 1327 (1990).

13. J. Tanaka, T. Kishi, and M. Tanaka, Bull. Chem. Soc. Jpn. 47, 2376 (1974).

14. T. J. Kosic, C. L. Schosser, and D. D. Dlott, Chem. Phys. Lett. 96, 57 (1983).

15. S. De Silvestri, J. G. Fujimoto, E. P. Ippen, E. B. Gamble, L. R. Williams, and K. A. Nelson, Chem. Phys. Lett. 116, 146 (1985).

16. R. L. Fork, B. I. Greene, and C. V. Shank, Appl. Phys. Lett. 38, 671 (1981); J. A. Valdmanis, R. L. Fork, and J. P. Gordon, Opt. Lett. 10, 131 (1985).

17. W. H. Knox, M. C. Downer, R. L. Fork, and C. V. Shank, Opt. Lett. 9, 552 (1984).

18. A. M. Weiner, J. P. Heritage, R. J. Hawkins, R. N. Thurston, E. M. Kirschner, D. E. Leaird, and W. J. Tomlinson, Phys. Rev. Lett. 61, 2445 (1988).

19. A. M. Weiner and D. E. Leaird, Opt. Lett. 15, 51 (1990).

20. Y.-X. Yan and K. A. Nelson, J. Chem. Phys. 87, 6240, 6257 (1987).

21. Y. J. Yan and S. Mukamel, J. Chem. Phys. 94, 997 (1991).

22. J. P. Heritage, R. N. Thurston, W. J. Tomlinson, A. M. Weiner, and R. H. Stolen, Appl. Phys. Lett. 47, 87 (1985).

23. A. M. Weiner, D. E. Leaird, G. P. Wiederrecht, M. J. Banet, and K. A. Nelson, in Picosecond and Femtosecond Spectroscopy from Laboratory to Real World, K. A. Nelson, ed., Proc. Soc. Photo-Opt. Instrum. Eng. 1209, 185 (1990). 